\title{
Induced symmetry breaking and a new phase of hadronic matter at high density
}

\author{
K. Langfeld ${ }^{\mathrm{a}}$ \\ anstitut für Theoretische Physik, Universität Tübingen, \\ Auf der Morgenstelle 14, D-72076 Tübingen
}

The notion induced symmetry breaking (ISB) is introduced as a generalization of the spontaneous symmetry breaking mechanism and is illustrated in a simple two flavor spin model. In the case of QCD at finite baryon density, I argue that the quark interaction induced by zero sound satisfies the prerequisites which are necessary for a ISB scenario. In this scenario, the quark condensate sharply drops at the critical value of the chemical potential in coincidence with a rapid increase of the baryon density. The spectrum of the light particles is discussed below and above this phase transition. The consequences of the ISB mechanism for heavy ion collisions are briefly addressed.

\section{Introduction}

The understanding of low energy QCD requires powerful non-perturbative techniques. Such a technique was already outlined by Goldstone in the early sixties [1,2]. He pointed out that the vacuum structure does not necessarily respect the symmetries of the Lagrangian. In this event, the symmetry is spontaneously broken and massless excitations exist which carry the quantum numbers of the generators corresponding to the spontaneously broken. In particular, assuming that the chiral symmetry of QCD is spontaneously broken, one understands the particular role of the light pseudo-scalar mesons in the spectrum without solving the full strong coupling problem.

The efforts to understand the evolution of the universe [3] or compact star matter [4] has posed a new challenge, the importance of which has steadily grown in the last years: the properties of hadrons at intermediate and high baryon density. This subject has attracted recent interest due to the data of the CERES and the HELIOS groups, who reported deviations of heavy ion collision data from the theoretical predictions according to present understanding [5,6]. They find that the dilepton yield in Au-Au collisions is enhanced in the invariant mass range of $300 \ldots 600 \mathrm{MeV}$ compared with the collisions $\mathrm{p}-\mathrm{Be}$ and $\mathrm{p}-\mathrm{Au}$ [5,6]. The experimental data have launched a vivid discussion on the origin of the dilepton enhancement. Most proposals focus on the role of the in medium $\rho$-meson, which dominantly couples to photons. One finds a good agreement of theory and experiment in $\mathrm{S}-\mathrm{Au}$ collisions, if a in medium $\rho$ mass-shift [7] towards smaller values is included in the relativistic transport calculations [8.9]. It was subsequently observed that a broadening of the $\rho$-width in baryonic matter also results in a dilepton enhancement 
compatible with the accuracy of the present experimental data [10 12]. Both explanations are so far compatible with the prediction of QCD sum rules [13, 12.

In this paper, I will employ the powerful concept of symmetry breaking to study QCD baryon matter. The notion of induced symmetry breaking (ISB) is introduced with the help of a simple two flavor spin model. I will discuss the prerequisites which are necessary for the ISB scenario to occur in the case of QCD at finite chemical potential, and will argue that an effective quark theory the interactions of which are mediated by zero sound satisfies these conditions. In this case, a new state of hadronic matter occurs above a critical value of the chemical potential. In this new phase, a light vector meson emerges which dominantly couples to dileptons. The new state of matter might play a role for the dilepton enhancement actually observed in the CERES and HELIOS experiments.

\section{The concept of induced symmetry breaking}

Let us study the basic features of the ISB scenario in a simple spin model from solid state physics. For this purpose, we will briefly study a liquid consisting of two (classical) spins, one of unit length ("slow" degree of freedom), $\vec{s}_{l}, \vec{s}_{l}^{2}=1$, and the other spin $\vec{s}_{f}$ with fixed length $\left|s_{f}\right|<1$ and high mobility ("fast" degree of freedom). The two different types of spins possess a quadrupol type interaction implying that the screening and antiscreening, respectively, of the "slow" degrees of freedom by the "fast" ones is not biased (for zero magnetic field). The "slow" degree of freedom $\vec{s}_{l}$ is dressed by a cloud of spins $\vec{s}_{f}$. For an effective description of the "slow" degrees of freedom, it is therefore convenient to introduce the notion of a constituent spin $\vec{\phi}$. $\vec{\phi}$ is a collective degree of freedom composed of $\vec{s}_{l}$ and $\vec{s}_{f}$, averaged over $\vec{s}_{f}$. Fluctuations of the $\vec{s}_{f}$-cloud introduces a variable length for the constituent spin $\vec{\phi}$. Within the framework of this effective approach to the two-flavor spin liquid, the partition function,

$Z=\int\left[d \Omega_{x}\right] \exp \{-\beta H\}$

is a functional integral over the angular degrees of freedom $\Omega_{x}$ of $\vec{s}_{l}$. In order to be precise, I confine myself to 3 -dimensional vectors. I propose the following effective Hamiltonian,

$H=-\sum_{\langle x y\rangle} \vec{\phi}_{x} \vec{\phi}_{y}+V\left(\vec{\phi}^{2}\right)-\sum_{x} \vec{\phi}_{x} \vec{B}, \quad V\left(\vec{\phi}^{2}\right)=\lambda \vec{\phi}^{2}\left(\vec{\phi}^{2}-2\right)$,

where the "constituent" spins $\vec{\phi}$ are located at the lattice sites $x$, and where the sum over $\langle x y\rangle$ extends over nearest neighbors. The effective length of the "constituent" spins $\vec{\phi}$ is mainly controlled by the potential $V\left(\vec{\phi}^{2}\right)$ and can be obtained from $\langle H\rangle_{\Omega} \rightarrow$ minimum. The potential $V\left(\vec{\phi}^{2}\right)$ possesses its global minimum at $\vec{\phi}^{2}=1$, where the curvature of the potential at the minimum is governed by $\lambda$. The fields $\vec{\phi}_{x}$ interact with a constant external "magnetic" field $\vec{B}$. We assume that $\lambda$ is independent of the magnetic field in the range of interest. In the limit $\vec{B}=0$, the model defined by (2) is invariant under a global rotation of the vectors.

The theory (2) of the constituent spin can be easily studied employing numerical simulations. It turns out that the mean field approach reproduces the gross features. For 


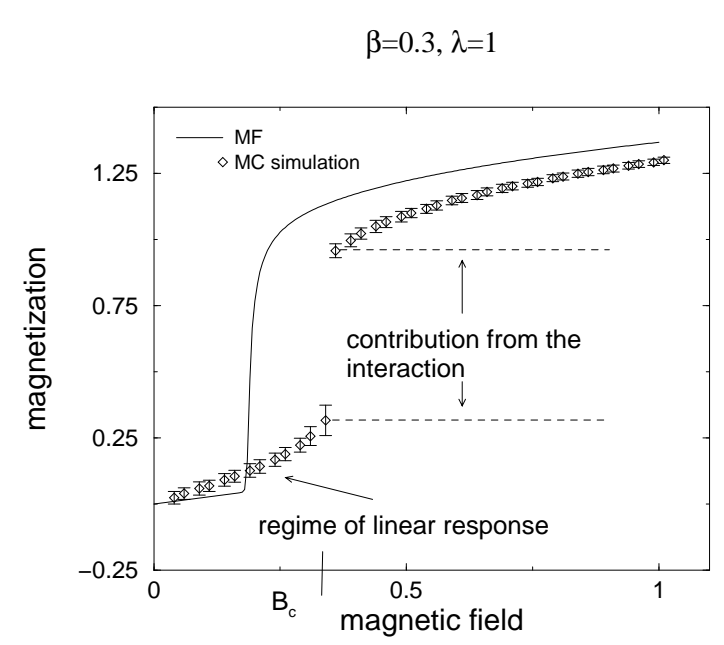

Figure 1. The magnetization as function of the external magnetic field for an inverse temperature $\beta=1 / T=0.3<1 / T_{\text {Curie }}$. $\beta=0.3, \lambda=1$

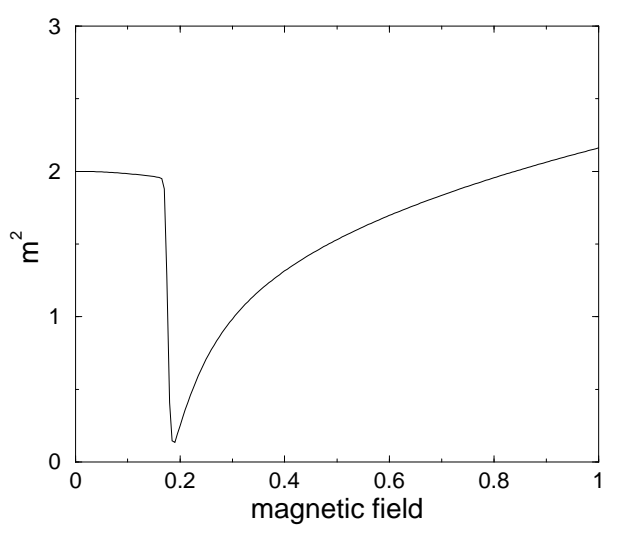

Figure 2. The behavior of the screening mass as function of the external magnetic field (MF-approximation).

$\lambda \gg 1$, the model (2) is reduced to an Heisenberg spin model. In this case and for temperatures larger than the Curie temperature, the magnetization monotonically increases with the external magnetic field. For $\lambda \approx 1$, the spin system shows the ISB behavior: for values of the magnetic field smaller than the critical value $B_{c}$, one observes a linear response of the magnetization with increasing external field. At the critical value $B_{c}$, a rapid increase of the magnetization takes place (see figure 11).

This phenomenon of ISB can be understood as follows: since the interaction of "slow" and "fast" degrees of freedom does not prefer screening or anti-screening, the average length of the constituent spin is of order unity at zero magnetic field. At non-zero magnetic field, both $\vec{s}_{l}$ and $\vec{s}_{f}$ are partially aligned along the direction of the magnetic field and the length of the constituent spins is shifted towards larger values on average. Finally, the residual interaction of the constituent spins is large enough for a spontaneous breaking of rotational symmetry and induced symmetry breaking takes place.

Since the interaction provides a large contribution to the order parameter, i.e. the magnetization, in addition to the (small) contribution from the external magnetic field, one expects the presence of long range correlations by virtue of Goldstone's theorem. In order to reveal these correlations, it is convenient to define the screening mass $m$ by

$\frac{1}{m^{2}} \delta_{i k}=\int d^{3} x\left\langle\phi_{i}(\vec{x}) \phi_{k}(0)\right\rangle=\frac{\partial \mathcal{M}_{i}}{\partial B_{k}}$

where $\mathcal{M}_{i}:=\left\langle\phi_{i}\right\rangle$ is the magnetization and $\vec{B}$ is the constant external magnetic field. Figure 2 shows the dependence of the screening mass on the external magnetic field. At the critical value $B_{c}$, one observes a sudden decrease of $m$ corresponding to long range correlations. Note that the excitations are not strictly massless due to the explicit breaking 
of rotational symmetry by the external field $\vec{B}$. For values $B>B_{c}$, the screening mass increases. Finally, the concept of an approximate symmetry becomes meaningless for a strong explicit symmetry breaking, i.e. $B \gg B_{c}$.

\section{ISB and QCD at finite chemical potential}

\subsection{Prerequisites for ISB}

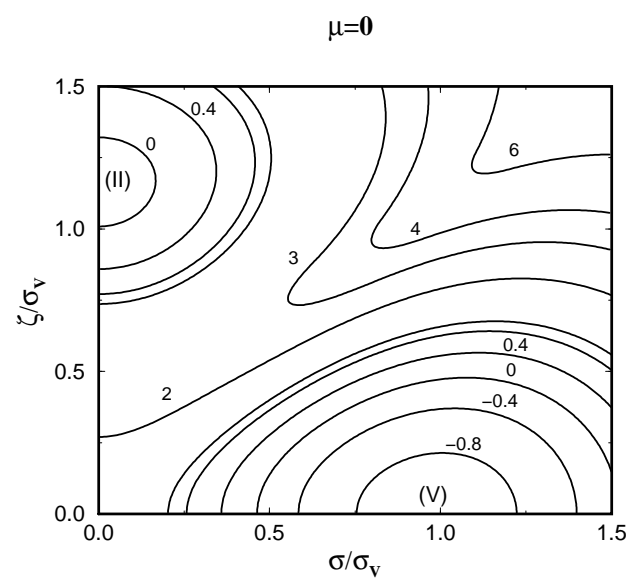

Figure 3. Lines of constant effective potential as function of the quark condensate $\sigma$ and the baryon density $\zeta$ at zero chemical potential.

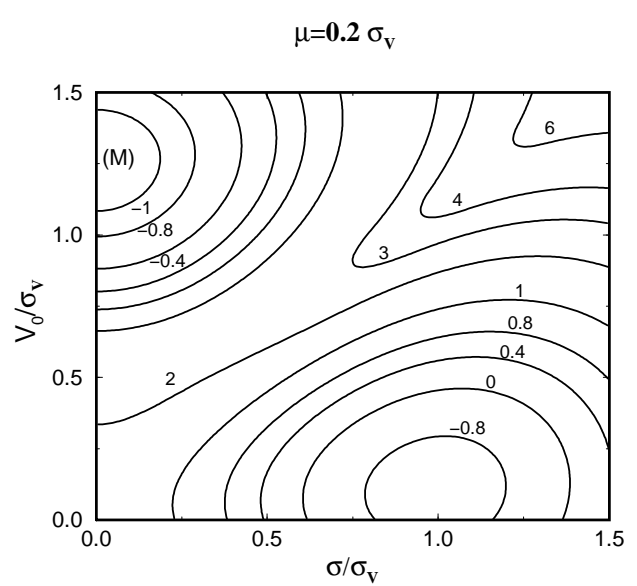

Figure 4. Same as figure 3 at a finite value of the chemical potential.

The sole condition for ISB to occur in hadron matter [14] can be most easily anticipated from the effective potential as function of the quark condensate $\sigma=\langle\bar{q} q\rangle$ and the baryon density $\zeta=\left\langle\bar{q} \gamma_{0} q\right\rangle$ as sketched in figure 3. Whereas the global minimum at $(\sigma, \zeta)=\left(\sigma_{v}, 0\right)$ constitutes the vacuum, the crucial assumption is that a second, local minimum, i.e. II in figure 3 , occurs at $(0, \zeta \neq 0)$. At finite values of the chemical potential $\mu$, this second minimum $I I$ gets more pronounced than the state $V$, since $\mu$ acts as a Lagrange multiplier for the baryon density, whereas the impact of $\mu$ on the quark condensate is of indirect nature. Increasing the chemical potential $\mu$, the generic behavior of the effective potential (at the axis) is illustrated in figure 5. At $\mu<\mu_{c}$, the non-vanishing values of the quark condensate signals a spontaneous breakdown of chiral symmetry. For $\mu>\mu_{c}$, the state $I I$ becomes the true ground state. Chiral symmetry is restored $(\sigma \approx 0)$, and the interaction strongly contributes to baryon density $\zeta$. ISB as discussed in the previous section sets in.

The change of the state structure at $\mu=\mu_{c i}$ tremendously influences the particle spectrum: firstly, we expect from Goldstone's theorem the presence of a light particle which carries the quantum numbers of a flavor singlet (non-relativistic) vector meson [14]. On the other hand, chiral symmetry is restored, and the pion ceases to play a special role as the lightest hadron. We therefore expect its mass in the range of several hundred 


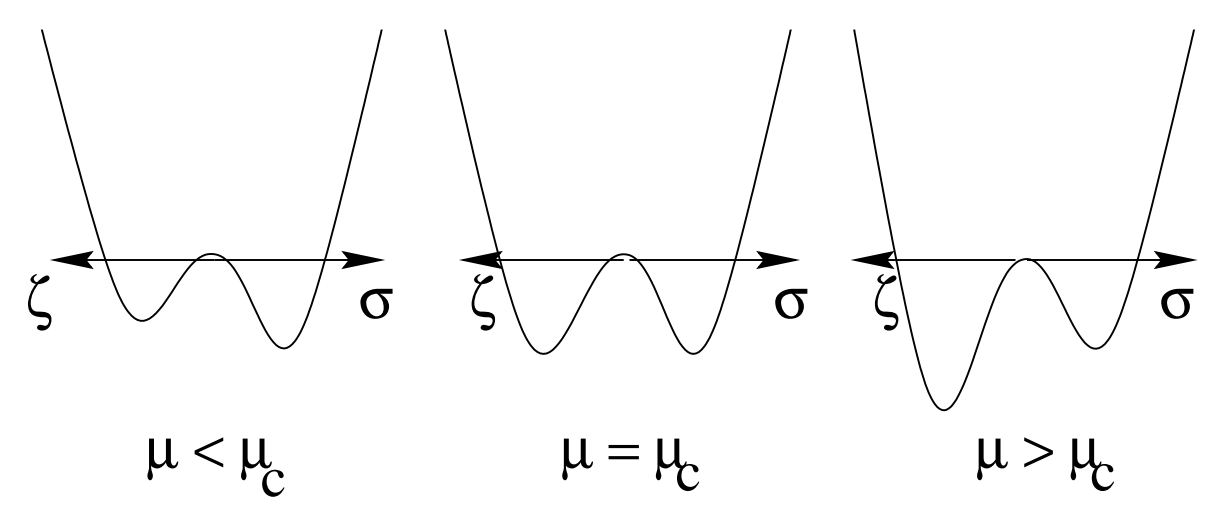

Figure 5. Schematic plot of the effective potential at the axis $(\sigma, 0)$ and $(0, \zeta)$ for several values of the chemical potential $\mu$.

MeVs. The properties of the emerging vector particle were studied in [14] in great detail. In particular, one expects from Goldstone's theorem that this vector particle dominantly couples to dilepton pairs. We were able to derive a Gell-Mann-Oakes-Renner type of relation [14. Our formula

$m_{V}^{2} f_{V}^{2}=2 \mu\left\langle\bar{q} \gamma_{0} q\right\rangle$

relates the mass of the vector particle $m_{V}$ and its decay constant $f_{V}$ to the chemical potential $\mu$ and the baryon density. Relation (国) quantifies the influence of the small explicit (on top of the spontaneous) breaking of Lorenz invariance via the chemical potential.

\subsection{Quark interactions mediated by zero sound}

The above scenario at finite density does not invoke any particular quark model, but relies on the existence of the local minimum $I I$. Whether this minimum exists in the case of QCD is up to now an open question. In the following, I will argue that the quark interactions induced by zero sound may meet the prerequisites for an ISB scenario. For this purpose, we studied the following effective quark theory [14]

$$
\begin{aligned}
Z\left[s, j_{\mu}\right] & =\int \mathcal{D} q \mathcal{D} \bar{q} \mathcal{D} \sigma \mathcal{D} \pi \mathcal{D} V_{\mu} e^{-\int d^{4} x\left[L-s(x) \sigma(x)-j_{\mu}(x) V^{\mu}(x)\right]} \\
L & =\bar{q}(x)\left(i \not \partial-\sigma(x)+i \gamma_{5} \pi(x)+i V_{\mu}(x) \gamma^{\mu}\right) q(x) \\
& +\frac{N}{2} g_{s}\left[(\sigma(x)-m)^{2}+\pi^{2}(x)\right] \\
& +\frac{N}{2}\left\{V_{\mu}(x)\left(-\partial^{2} \delta_{\mu \nu}+\partial_{\mu} \partial_{\nu}\right) V_{\nu}(x)+m_{v}^{2}\left[\left(V_{0}(x)-\mu\right)^{2}+V_{k}^{2}(x)\right]\right\}
\end{aligned}
$$

where $N$ is the number of colors. $\sigma$ and $\pi$ denote the usual scalar and pionic hadron fields. In addition to these fields, a vector-like quark interaction is mediated by the field $V_{\mu}(x)$. Note that the coupling of these vector fields to the quark is defined in such a way that the zero component $V_{0}$ acts like a chemical potential. Note that this is different from the standard coupling of $\rho$ and $\omega$ mesons to quarks. Fluctuations of $V_{0}$ act like fluctuations in the chemical potential. At the level of the effective quark theory (5), 
the vector field $V_{\mu}$ therefore describes a vector type quark interaction due to density fluctuations, which is present on top of the interactions mediated by the mesons $\sigma, \pi$, $\rho, \omega$. The introduction of the vector field $V_{\mu}$ is reminiscent of the solid state physics phenomenon of zero sound [15], which can be observed in Fermi liquids. Whereas "first sound" describes density fluctuations which are due to distortions of the thermodynamical equilibrium, zero sound can be understood as vibration of the Fermi sphere and propagates due to the presence of the quasi-particle interaction.

The effective quark theory (5:6) was studied in [14] in great detail employing standard techniques. The resulting effective potential was already shown in figure 3 and 1 , which were used for illustration purposes before.

Acknowledgments: It is a pleasure to thank my collaborators Mannque Rho and Hugo Reinhardt. I thank Niels Schopohl for interesting discussions on Fermi liquids, and Michael Baake for helpful information in the same context. I am indebted to H. Reinhardt for support.

\section{REFERENCES}

1. J. Goldstone, Nuovo Cimento 19 (1961) 154;

J. Goldstone, A. Salam, S. Weinberg, Phys. Rev. 127 (1962) 965.

2. F. J. Yndurain, 'Quantum Chromodynamics', Springer Verlag, 1983.

3. see e.g. E. W. Kolb, M. S. Tuner, The Early Universe, Addison-Wesley 1990.

4. G. E. Brown and M. Rho, Phys. Rept. 269 (1996) 333.

5. CERES collaboration, Phys. Rev. Lett. 75 (1995) 1272; Nucl. Phys. A590 (1995) $103 \mathrm{c}$.

6. HELIOS-3 collaboration, Nucl. Phys. A590 (1995) 93c.

7. G. E. Brown, M. Rho, Phys. Rev. Lett. 66 (1991) 2720.

8. G. Q. Li, C. M. Ko, G. E. Brown, Phys. Rev. Lett. 75 (1996) 4007; Nucl. Phys. A606 (1996) 568.

9. C. M. Ko, G. Q. Li, G. E. Brown, H. Sorge, Nucl. Phys. A610 (1996) 342c.

10. R. Rapp, G. Chanfray, J. Wambach, Phys. Rev. Lett. 76 (1996) 368; Nucl. Phys. A617 (1997) 472.

11. C. Song, V. Koch, S. H. Lee, Phys. Lett. B366 (1996) 379.

12. F. Klingl, N. Kaiser, W. Weise, Nucl. Phys. A624 (1997) 527.

13. T. Hatsuda, S. H. Lee, Phys. Rev. C46 (1992) R34; T. Hatsuda, S. H. Lee, H. Shiomi, Phys. Rev. C52 (1995) 3364; T. Hatsuda, nucl-th/9702002.

14. K. Langfeld, H. Reinhardt, M. Rho, Nucl. Phys. A622 (1997) 620.

15. E. M. Lifschitz, L. P. Pitajewski, Landau-Lifschitz, vol 10, chap.8; A. L. Fetter, J. D. Walecka, Quantum theory of many particle systems, McGraw-Hill 1971. 